\title{
Antitubercular Drug induced Hepatitis: An Adverse Drug Reaction
}

\author{
Aisha Begum*, Uzma Parveen, Safura Sultana, Nausheen Fatima, Mohd Fareedullah, Zareenunnisa \\ Department of Pharmacy Practice, Deccan School of Pharmacy, Hyderabad, Telangana, INDIA.
}

\begin{abstract}
Antitubercular drugs are the first line agents for the management of tuberculosis. Drug induced hepatotoxicity is an unexceptional adverse effect mostly caused by antitubercular drugs leading to cessation of therapy. The keystone for the treatment of tuberculosis is a 6-month regimen consisting of drugs such as Isoniazid, rifampicin, pyrazinamide and ethambutol. Although Antitubercular drugs are only option for the treatment for pulmonary TB, Hepatotoxicity is one of the most detrimental adverse events leading to interruption of treatment thereby causing drug resistance. In this case the patient was recently diagnosed with pulmonary tuberculosis one month ago, consequently developed hepatotoxicity due to use of anti-tuberculosis drugs. Naranjo Adverse Drug Reaction Probability Scale was used to assess the adverse Drug effect and the score was found to be probable thereby indicating that Anti TB drugs cause hepatitis.
\end{abstract}

Key words: Anti-tuberculosis drugs, Hepatotoxicity, Pulmonary TB, Adverse effect, Drug resistance.

\section{INTRODUCTION}

Tuberculosis has been proclaimed as a worldwide health emergency as stated by World health organization (WHO) nearly one third of world population was postulated to be affected with TB. ${ }^{1}$ According to RNTCP (revised national tuberculosis control program, DOTS is the mainstay employed for the management of tuberculosis. ${ }^{2}$ Drug induced liver injury is a severe detrimental effect caused by antitubercular drugs which not only leads to diminution of efficacy but also leads to high rise in morbidity and mortality. ${ }^{3}$ Presently approved first line therapy for treatment of TB includes Isoniazid, Rifampicin, Pyrazinamide and Ethambutol for 2 months succeeded by 4 months of isoniazid and rifampicin ${ }^{4}$ The toxicity symptoms noticeable are in the form of nausea, vomiting, yellowish discoloration of eyes. ${ }^{5}$ Numerous risk factors contributing to ATT induced toxicity are venerable age, sex, improper use of drugs, liver diseases, viral infection due to HBV, HCV and HIV virus, advanced $\mathrm{TB}$, asian ethnicity etc. ${ }^{6}$

\section{CASE PRESENTATION}

A 65-year-old patient presented to the tertiary care hospital with chief complaints of shortness of breath grade III, productive cough with thick sputum, fever, sore throat, hoarseness of voice in the last 1 month. Physical examination was done. Diagnostic test such as chest $\mathrm{X}$ ray, $\mathrm{ZN}$ staining were performed. A chest $\mathrm{X}$ ray depicted the presence of Lower lobe consolidation, sputum smear was positive for acid fast bacilli. She was diagnosed as pulmonary tuberculosis and was on anti TB drug treatment for 1 month. She was on Isoniazid $75 \mathrm{mg}$, Rifampicin $150 \mathrm{mg}$ Pyrazinamide 400 $\mathrm{mg}$, Ethambutol $275 \mathrm{mg}$. The symptoms improved initially, however on $25^{\text {th }}$ day she was admitted in the pulmonology department with chief complaints of jaundice for 3 days, pain in abdomen, Diarrhea, itching over lower limbs.

On enquiring the patient, she had a history of hypertension and Hypothyroidism, however there was no past history of any surgeries contact with the TB patient and blood transfusion. Additionally, she denied devouring any herbal and dietary supplements. Anti-tuberculosis drugs were immediately withdrawn upon admission to
DOI: 10.5530/ijopp.13.3.47

Address for correspondence: Aisha Begum, Department of Pharmacy Practice, Darussalam, Aghapura, Hyderabad 500001, Telangana, INDIA.

Phone no: +91 8919577252 Email Id: shinenoor58@gmail. com

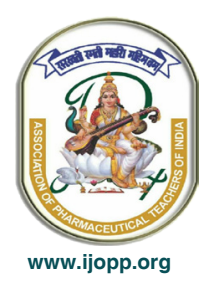




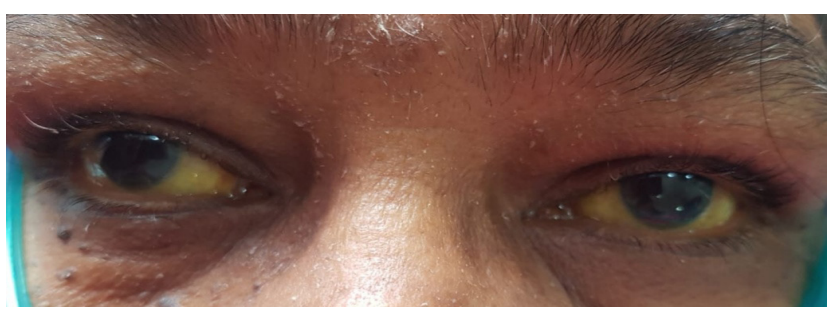

Figure 1: Yellowish eyes (jaundice) due to elevated liver enzymes.

the hospital as the clinical and laboratory investigations confirmed the diagnosis as Anti tubercular drug induced hepatitis.

Physical examination of the patient displayed pallor and icterus. Dermatology opinion revealed generalized xerosis of skin, no erythematous or papular or any other significant lesions were observed. As it is known that anti TB drugs ADR include hepatotoxicity, ATT was stopped for 3 days and hepatoprotective agents such as Ursodeoxycholic acid and symptomatic treatment was provided as the Patient condition was improving as evident by liver function test, the second line agents of Anti TB therapy Amikacin and Levofloxacin along with Ethambutol was again started on fourth day of admission. Laboratory findings demonstrated lymphopenia, (14.6\%), followed by increased Total Protein $(7.1 \mathrm{gm} / \mathrm{dl})$, Total Bilirubin (11.6\%), Direct Bilirubin $(9.5 \mathrm{gm} / \mathrm{dl})$, decreased Albumin $(2.5 \mathrm{gm} / \mathrm{dl})$, elevated liver enzymes such as SGOT (490U/I), SGPT (206 U/I), Alkaline Phosphatase $(136 \mathrm{U} / \mathrm{I})$. Serum creatinine was slightly elevated. Viral markers such as Hepatitis E virus was positive.

Chest X-ray showed left lower apical segment, irregular cavity lesion, centrilobular nodular opacity plus tree in bud opacities in left lateral and basal segments. USG of abdomen and pelvis revealed cholelithiasis. The patient was managed accordingly by using analgesics, antipyretics, hepatoprotective agents, empirical antibiotics, emollients, moisturizing creams, $2^{\text {nd }}$ line Anti-tubercular drugs. The laboratory reports returned to normal at the time of discharge. Upon follow up the patient was tolerating $2^{\text {nd }}$ line Anti-tubercular drugs effective.

According to Paracelsus the father of toxicology "Dose makes the poison" is more appropriate in case of drug induced hepatotoxicity. ${ }^{7}$ Reports published by WHO indicates 21000 liver transplantation in the year 2007. Therefore protecting the liver should be ultimate goal. It is predicted that Anti-TB drug induced hepatitis occurs in approximately $28 \%$ of cases. $^{7}$

Anti-TB treatment is the most effective method of controlling the spread of the disease, however adverse events that occurs occasionally interrupts the treatment.
The various types of adverse events of Antitubercular drugs includes hepatotoxicity, acute kidney injury, gastrointestinal discomfort, rash, a drug fever and optic neuritis. The first line agents for Anti TB treatment are four drugs Isoniazid, Rifampicin, Pyrizinamide and Ethambutol among which Isoniazid, Rifampicin and Pyrizinamide are primarily metabolized by liver therefore these drugs from the main cause of hepatotoxicity in case of drug induced hepatotoxicity during anti TB treatment. ${ }^{8}$

The risk factors for Anti TB drug induced hepatotoxicity are as follows geriatric patients, female gender, under nourishment, Ethanolic, history of liver diseases, lung parenchymal and HIV infection. However, the exact mechanism of biochemical reaction and pathogenesis of Anti TB drug induced hepatotoxicity remains uncertain. ${ }^{8}$ Liver is an important body organ according to physiological point of view as it is responsible for metabolism of many xenobiotics. The incidence of hepatotoxicity is $2.6 \%$ with combination of Isoniazid and Rifampicin, $1.1 \%$ with Rifampicin monotherapy and $1.6 \%$ with isoniazid monotherapy. ${ }^{9}$ We are reporting a case of 65 years old female patient who has been diagnosed with TB one month back when she was admitted with the respiratory distress as chief complaint. The patient was started with DOT therapy of ATT to which she developed jaundice and abnormality in LFT's and was again diagnosed as ATT induced hepatitis. As it is known that anti TB drugs ADR include hepatotoxicity, AT'T was stopped for 3 days and hepatoprotective agents such as Ursodeoxycholic acid and symptomatic treatment was provided as the Patient condition was improving as evident by liver function test, the second line agents of Anti TB therapy Amikacin and Levofloxacin along with Ethambutol was again started on fourth day of admission. As known that there is no drug free from ADR but first line agents Isoniazid, Rifampicin, Pyrazinamide causes serious adverse drug reactions which can also be fatal in some cases. In this patient the second line treatment was effective and patient regained to normal health status.

The formula $\mathrm{R}=$ ALT/ULN divided by ALP /ULN given by American College of Gastroenterology (ACG) clinical guidelines can be used to identify the various types of drug induced Liver injury (DILI): Acute hepatocellular $(\mathrm{R}>5)$, Mixed $(\mathrm{R}=2-5)$ or cholestatic $(\mathrm{R}<2) .{ }^{10}$ In addition, the current clinical guidelines given by CDC AIS and the Infectious Disease Society of America should be followed that states that patients treated with Isoniazid and associated with certain risk factors should be screened once in a month for detecting any adverse event Liver Enzymes such as Transaminases should be accounted before start of therapy and then after every 4weeks between the ongoing treatment. ${ }^{11}$ There can be several 
complications with ADRs of Anti tubercular therapy, the first step would be cessation of therapy which will has its various implications such as increased morbidity, mortality rate, more outpatient visits and increased hospitalization. ${ }^{12}$ So our treatment should be more specific, focused and newer medical methodology should be applied to prevent drug induced hepatotoxicity.

\section{CONCLUSION}

Although Antitubercular drugs are only option for the treatment for pulmonary TB, Hepatotoxicity is one of the detrimental adverse events leading to interruption of treatment thereby causing drug resistance. Among the first line Anti-TB drugs Rifampicin is the most common cause of hepatotoxicity in comparison with Isoniazid and pyrazinamide. Consequently $2^{\text {nd }}$ line alternatives such as levofloxacin are used as substitute to minimize hepatotoxicity. The patient on Anti TB treatment should be counselled by the pharmacist about the risk of hepatotoxicity, should be screened for susceptibility based on the presence of certain risk factors and monitoring of signs and symptoms to withdraw the medication. Clinician should also perform therapeutic drug monitoring (TDM) in case of drug resistant TB, drug -drug interaction, slow response to treatment or several underlying diseases is present.

\section{ACKNOWLEDGEMENT}

We take this valuable opportunity to convey our gratitude to Dr. S.A Azeez, Principal, Deccan School of Pharmacy and Mohd. Fareedllah, Associate Professor of Deccan School of Pharmacy for granting essential facilities, suggestions and inspiration.

\section{ABBREVIATIONS}

DOTS: Direct observed treatment; LFT: Liver Function test; HBV: Hepatitis B virus; HCV: Hepatitis C virus; HEV: Hepatitis E virus; AST: Aspartate amino transferase; ALT: Alanine amino transferase.

\section{REFERENCES}

1. Brewer TF, Heymann SJ. To control and beyond: moving towards eliminating the global tuberculosis threat. Journal of Epidemiology and Community Health. 2004;58(10):822-5.

2. Saha A, Shanthi FXM, Winston AB, Das S, Kumar A, Michael JS, Balamugesh T. Prevalence of hepatotoxicity from antituberculosis therapy: A five-year experience from South India. Journal of Primary Care and Community Health. 2016;7(3):171-4.

3. Gupta AK, Bala M. ATT-induced Hepatotoxicity: Culprit Drug. The Journal of the Association of Physicians of India. 2018;66(9):104.

4. Jeong I, Park JS, Cho YJ, Yoon HI, Song J, Lee CT, et al. Drug-induced hepatotoxicity of anti-tuberculosis drugs and their serum levels. Journal of Korean Medical Science. 2015 ;30(2):167-72.

5. Hussain Z, Kar P, Husain SA. Antituberculosis drug-induced hepatitis: Risk factors, prevention and management. Indian J Exp Biol. 2003;41(11):1226-32.

6. Sharma SK, Singla R, Sarda P, Mohan A, Makharia G, Jayaswal A, et al. Safety of 3 different reintroduction regimens of antituberculosis drugs after development of antituberculosis treatment-induced hepatotoxicity. Clinical Infectious Diseases. 2010;50(6):833-9.

7. Dey PR, Saha MR, Sen AR. An overview on drug-induced hepatotoxicity. Asian J Pharm Clin Res. 2013;6(4):1-4.

8. Sakashita K, Murata K, Takahashi Y, Yamamoto M, Oohashi K, Sato Y, et al. A case series of acute kidney injury during anti-tuberculosis treatment. Internal Medicine. 2019;0813-18.

9. Yew WW, Leung CC. Antituberculosis drugs and hepatotoxicity. Respirology. 2006;11(6):699-707.

10. Kabbara WK, Sarkis AT, Saroufim PG. Acute and fatal isoniazid-induced hepatotoxicity: A case report and review of the literature. Case Reports in Infectious Diseases. 2016.

11. Li AA, Dibba P, Cholankeril G, Kim D, Ahmed A. Case Report of IsoniazidRelated Acute Liver Failure Requiring Liver Transplantation. Diseases. 2018;6(2):40.

12. Yee D, Valiquette C, Pelletier M, Parisien I, Rocher I, Menzies D. Incidence of serious side effects from first line antituberculosis drugs among patients treated for active tuberculosis. American Journal of Respiratory and Critical Care Medicine. 2003;167(11):1472-7. 